\title{
Somatic JAK-2 V617F Mutational Analysis in Polycythemia Rubra Vera: a Tertiary Care Center Experience
}

\author{
Sadia Sultan ${ }^{1 *}$, Syed Mohammed Irfan ${ }^{1}$, Sadia Rashid Khan ${ }^{2}$
}

\begin{abstract}
Background: Polycythemia rubra vera (PV), being a primary polycythemia, is caused by neoplastic proliferation of erythroid, megakaryocytic and granulocytic lineages which result in panmyelosis. PV patients have a somatic acquired mutation in the Janus kinase (JAK2) pathway, rendering cell proliferation independent of the normal regulatory mechanisms that regulate erythropoiesis. The rational of this study was to determine the prevalence of the JAK-2 V617F mutation in Pakistani patients with PV. Materials and Methods: In this cross sectional study, 26 patients with PV were enrolled from January 2010 to December 2014. Patients were diagnosed based on WHO criteria for PV. All were screened for G-T point mutation (V617F) in the JAK2 gene on chromosome 9 by an allele specific PCR. Results: The mean age was 53.4 \pm 9.31 years (range 36-72) and the male to female ratio was 2:1. The frequency of JAK2 V617F positivity in our PV patients was found to be $92.3 \%$. Overall $30.7 \%$ of patients were asymptomatic and remaining $69.3 \%$ presented with symptomatic disease. The mean hemoglobin was $18.1 \pm 1.9 \mathrm{~g} / \mathrm{dl}$ with the mean hematocrit of $55.6 \pm 8.3 \%$. The mean total leukocyte count was $12.8 \pm 7.1 \times 10^{9} / \mathrm{l}$ and the platelet count was $511 \pm 341.9 \times 10^{\circ} / \mathrm{l}$. A positive correlation of JAK2 $\mathrm{V} 617 \mathrm{~F}$ mutation was established with high TLC count $(P=0.01)$. No correlation of JAK2 V617F could be established with age or gender $(\mathrm{P}>0.05)$. Conclusions: The JAK2 V617F mutation frequency in our PV patients was similar to those reported internationally. Screening for the mutation in all suspected PV cases could be beneficial in differentiating patients with reactive and clonal erythrocytosis.
\end{abstract}

Keywords: JAK-2 V617F - polycythaemia rubra vera - erythrocytosis - Pakistan

Asian Pac J Cancer Prev, 17 (3), 1053-1055

\section{Introduction}

Myeloproliferative neoplasms (MPN) have been previously called myeloproliferative disorders are chronic, clonal hematopoietic stem cell group of disorders characterized by enhanced proliferation of one or more myeloid lineage cells (Sag et al., 2015; Yang et al., 2015). Polycythaemia rubra vera (PV) is a indolent MPN characterized by an absolute increase in the number of red blood cells, usually accompanied by leukocytosis, thrombocytosis and splenomegaly (Ma et al., 2008; Zhang et al., 2014).

The diagnosis of PV has always been challenging previously. Since the parameters as defined by Polycythemia Vera Study Group for diagnosis are extensive and expensive, which make difficultly in diagnostic certainty. In 2005, researchers identified a somatic acquired mutation in the JAK2 gene on chromosome 9 which has been shown to be associated with various myeloproliferative neoplasms (Baxter et al., 2005; James et al., 2005). This identified point mutation is a G-C to T-A transversion, resulting in the substitution of valine by phenylalanine at codon 617 (JAK2V617F) (Sazawal et al., 2010).
Majority of PV patients carry this acquired somatic gain of function mutation of the Janus associated kinase genes (Agarwal et al., 2015; Yonal-Hindilerden et al., 2015). JAK2 V617F has been recognized in varied subsets of each Ph-chromosome negative MPNs (Duangnapasatit et al., 2015). Various studies have demonstrated that this mutation is present in more than $95 \%$ of the patients with PV and in 55 and $65 \%$ of patients with ET and PMF respectively (Tefferi et al., 2015). Therefore, determination of JAK2V617F mutation is potentially diagnostic tool for PV patients.

We here sought to look for the prevalence of JAK2V617F mutation in Pakistani patients with polycythemia vera and also to determine its correlation with age, gender and hematological parameters.

\section{Materials and Methods}

This was a retrospective cross sectional study conducted at Liaquat National Hospital and Medical College, Karachi which is an over 700 bed tertiary care teaching institute, from January 2010 till December 2014.

Patients were diagnosed to had PV according to the 
World Health Organization (WHO) criteria (Thiele et al., 2008). Diagnosis required meeting both two major criteria and one minor criteria or the presence of first major criteria together with two minor criteria.

\section{Major criteria included}

i) Hemoglobin $>18.5 \mathrm{gm} / \mathrm{dl}$ in male or $>16.5 \mathrm{gm} /$ $\mathrm{dl}$ in women; ii) Demonstration of JAK2 V617F mutation;with minor criteria; iii) Bone marrow biopsy showing hypercellularity for age with panmyelosis; $i v$ ) Serum erythropoietin levels below the reference range from normal.

In vitro culture of erythroid colonies was not assessed as this facility is not available in our country. Complete blood counts were determined by Automated Cell Dyne Ruby Counter (Abott, Diagnostics). Blood sample of all the patients were taken in an Ethylene-diamine tetra acetate anticoagulant tube and PCR was performed for G-T point mutation (V617F) in the JAK2 gene on chromosome 9 by using allele specific primers. Bone marrow aspirate and trephine biopsy specimen were performed with Jamshidi needle, were evaluated by specialist hematopathologists. Serum erythropoietin was measured by ELISA technique through automated analyzer.

Approval from the institutional ethical review committee for the study was obtained prior to study.

\section{Statistical analysis}

Data was collected and analyzed using SPSS windows version 22 statistical package (SPSS Inc, Chicago, IL, USA). The results were expressed as mean \pm SD for quantitative variables and qualitative variables are presented as frequency and percentages. Student ' $t$ ' test was applied for the comparison of mean. Data were considered statistically significant at $P$ value $<0.05$. Chisquare test was applied to assess the correlation.

\section{Results}

A total of 26 confirmed Polycythaemia vera patients were included in this study. The mean age of patients was 53.42 \pm 9.31 years (range 36-72). Out of 26 patients, 17 were males $(65.3 \%)$ and 9 were females $(34.7 \%)$ with male to female ratio of $2: 1$.

Overall $18(69.3 \%)$ patients were symptomatic and remaining $8(30.7 \%)$ were diagnosed incidentally. In symptomatic patients, major complaints were headache (30.8\%); abdominal discomfort (23.07\%); blurred vision $(15.3 \%)$; pruritus $(11.5 \%)$ and vascular accident $(11.5 \%)$. The most common vascular event was transient ischemic attack $(7.6 \%)$, while one patient $(3.8 \%)$ presented with Budd-chiari syndrome. Physical examination revealed plethoric face and splenomegaly as predominant findings detected in $34.6 \%$ and $30.7 \%$ patients respectively.

The mean hemoglobin was $18.14 \pm 1.9 \mathrm{~g} / \mathrm{dl}$ (range 16.522.1 ) with the mean hematocrit of $55.6 \pm 8.3 \%$. The mean total leukocyte count was $12.8 \pm 7.1 \times 10^{9} / 1$ (range 4.3-28.6) and platelets counts of 511 $\pm 341.9 \times 10^{9} / 1$ (range 132-1462). Mean serum erythropoietin was $6.86 \pm 5.4 \mathrm{u} / \mathrm{mI}$.

JAK2 V617 F mutation was positive in $24(92.3 \%)$ patients out of total 26 study subjects. No correlation could be established between JAK2 V617F positivity with age and gender $(\mathrm{P}>0.05)$. However comparative analysis revealed significantly high total leukocytic count of $13.3 \pm 7.1$ in JAK2 V617F positive patients group as compared with negative group that was $7.3 \pm 3.2(\mathrm{P}=0.01)$.

\section{Discussion}

Polycythaemia vera belongs to clonal myeloproliferative neoplasms, exemplified by increased red cell proliferation, hyperviscosity, thromboembolism and intermittently overt bleed (Zhang et al., 2014; Tefferi et al., 2015). The present study detected the somatic JAK2 V617F mutation in $92.3 \%$ of Pakistani patients with PV.

There are limited studies reported from Pakistan on this hematopoietic malignancy (Khattak et al., 2012; Sadiq et al., 2013). It was noted that the prevalence of JAK2V617F mutation in our patients from southern Pakistan was comparable with that reported in the previous local studies (93.5\% and 100\%) from Pakistan (Khattak et al., 2012; Sadiq et al., 2013). JAK2 V617F positivity in PV has been observed from various racial backgrounds ranging from $80 \%$ to $100 \%$ (Karkucak et al., 2012; Sadiq et al., 2013). When compared with earlier reports, our results are intermediate with regional studies reported from India and Malaysia; $82 \%$ and $95.8 \%$ for JAK2V617F mutational expression respectively (Sazawal et al., 2010; Hamidah et al., 2012). Other studies from Turkey reported the frequency of JAK-2 positivity in $80 \%$ and $95 \%$ of patients with PV respectively (Karkucak et al., 2012; Payzin et al., 2014). Similarly, JAK2 mutation in Taiwanese patients with PV was detected in 28 out of 33 patients $(85 \%)$ (Lieu et al., 2008). The positivity rate for JAK 2 was $90.6 \%$ in one Iraqi study conducted on patients with polycythemia vera (Al-Thwani et al., 2010).

Compared with data from developed countries outcome are more or less similar. Recent study from China also reported the similar prevalence $(87.6 \%)$ in their patients that is comparable to our findings (Ren et al., 2015). Subsequently, Kiladjian et al. (2012) from France also reported mutational frequency as $95 \%$ in their study. Mutational frequency was determined as $88 \%$ for JAK2V617F in a large series from the European country (Duletic et al., 2012).

JAK2 V617F mutational expression in PV obviously segregates the disease into two broad categories. It has been also reported previously that PV cases with JAK-2 positivity displays a higher leucocyte count and haematocrit value as compared with negative phenotype (Zhu., 2011; Ren et al., 2015). These findings suggest that JAK-2 expression might reflect a sustained activation state of the hemopoeitic stem cell malignant clone.

One Chinese study reported significant correlation between high TLC counts and JAK-2 mutation in patients with polycythemia vera (Zhu., 2011). In the present study, significantly high total leukocytic count of $13.3 \pm 7.1$ was seen in JAK2 V617F positive patients group as compared with negative group that was $7.3 \pm 3.2(\mathrm{P}=0.01)$.

However in conflict to previous reports no correlation was established with high hemoglobin and JAK2 mutation in the present study (Ren et al., 2015). Also no correlation 
was established with age and gender. The findings need to be validated in study with the larger sample.

The smaller sample size and retrospective nature of study are our major limitations.

In conclusion, this study provides local informative facts, revealed that V617F mutations were present in the majority (92.3\%) of Pakistani patients with PV. Mutational screening for JAK2V617F by PCR should be incorporated into the initial evaluation of patients suspected to have polycythemia, which will reasonably distinguish between reactive and clonal erythrocytosis.

\section{References}

Agarwal MB, Malhotra H, Chakrabarti P, et al (2015). Myeloproliferative neoplasms working group consensus recommendations for diagnosis and management of primary myelofibrosis, polycythaemia vera, and essential thrombocythemia. Indian J Med Paediatr Oncol, 36, 3-16.

Al-Thwani AN, Yaseen NY, Khaleel KJ (2010). The Role of JAK2 Mutation in Polycythaemia Vera in Some Iraqi Patients. Iraqi J Cancer Med Genetics, 43, 42-5.

Baxter EJ, Scott LM, Campbell PJ, et al (2005). Acquired mutation of the tyrosine kinase JAK2 in human myeloproliferative disorders. Lancet, 365, 1054-61.

Duangnapasatit B, Rattarittamrong E, Rattanathammethee T, et al (2015). Clinical Manifestations and Risk Factors for Complications of Philadelphia Chromosome-Negative Myeloproliferative Neoplasms. Asian Pac J Cancer Prev, 16, 5013-8.

Duletic AN, Dekanic A, Hadzisejdic I, et al (2012). JAK2-v617F mutation is associated with clinical and laboratory features of myeloproliferative neoplasms. Coll Antropol, 36, 859-65.

Hamidah NH, Farisah NR, Azlinda AB, et al (2012). A study of JAK2 (V617F) gene mutation in patients with chronic myeloproliferative disorders. Clin Ter, 163, 109-13.

James C, Ugo V, Le Couedic JP, et al (2005). A unique clonal JAK2 mutation leading to constitutive signalling causes polycythaemia vera. Nature, 434, 1144-8

Karkucak M, Yakut T, Ozkocaman V, et al (2012). Evaluation of the JAK2-V617F gene mutation in Turkish patients with essential thrombocythemia and polycythemia vera. Mol Biol Rep, 39, 8663-7.

Kiladjian JJ (2012). The spectrum of JAK2-positive myeloproliferative neoplasms. Hematol Am Soc Hematol Educ Program, 2012, 561-6.

Khattak SA, Ahmed S, Anwar J, Bozdar M (2012). Frequency of Janus associated kinase 2 (V617F) mutation in patients of polycythemia vera. J Coll Physicians Surg Pak, 22, 80-3.

Lieu C, Wu H, Hon HS, et al (2008). Prevalence of the JAK2 V617F mutation in Taiwanese patients with chronic myeloproliferative disorders. Int Med J, 111, 145-9.

Ma X, Vanasse G, Cartmel B, Wang Y, Selinger HA (2008). Prevalence of polycythaemia vera and essential thrombocythemia. Am J Hematol, 83, 359-62.

Payzin KB, Savasoglu K, Alacacioglu I, et al (2014). JAK2 V617F mutation status of 232 patients diagnosed with chronic myeloproliferative neoplasms. Clin Lymphoma Myeloma Leuk, 14, 525-33.

Ren Y, Fu R, Qu W, et al (2015).Clinical analysis of 70 cases of polycythemia vera. Zhonghua Yi Xue Za Zhi, 95, 1378-81.

Sag SO, Gorukmez O, Ture M, et al (2015). MMP2 gene-735 C/T and MMP9 gene - $1562 \mathrm{C} / \mathrm{T}$ polymorphisms in JAK2V617F positive myeloproliferative disorders. Asian Pac J Cancer Prev, 16, 443-9.

Sazawal S, Bajaj J, Chikkara S, et al (2010). Prevalence of
DOI:http://dx.doi.org/10.7314/APJCP.2016.17.3.1053

JAK2 V617F Mutation in Polycythaemia Vera Cases in Pakistan

JAK2 V617F mutation in Indian patients with chronic myeloproliferative disorders. Indian J Med Re, 132, 423-7.

Sadiq MA, Ahmed S, Ali N (2013). Frequency of Janus associated kinase 2 (JAK2) mutation in patients of BCRABL negative myeloproliferative neoplasms. Applied Life Sciences, 2, 235-40.

Tefferi A, Barbui T (2015). Polycythaemia vera and essential thrombocythemia: 2015 update on diagnosis, riskstratification and management. Am J Hematol, 90, 162-73.

Thiele J, Kvasnicka HM, Orazi A, et al. Polycythaemia vera. In: Swerdlow HS, Campo E, Haris LN, et al (2008). WHO classification of tumours of haemopoietic and lymphoid tissues. Lyon: International agency for research on cancer, 40.

Yang JJ, Chen H, Zheng XQ, et al (2015). Methylated alteration of SHP1 complements mutation of JAK2 tyrosine kinase in patients with myeloproliferative neoplasm. Asian Pac J Cancer Prev, 16, 2219-25.

Yonal-Hindilerden I, Daglar-Aday A, Akadam-Teker B, et al (2015). The durden of JAK2V617F mutated allele in Turkish patients with myeloproliferative neoplasms. J Clin Med Res, 7, 161-70.

Zhang ZR, Duan YC (2014). Interferon apha $2 b$ for treating patients with JAK2V617F positive polycythaemia vera and essential thrombocytosis. Asian Pac J Cancer Prev, 15, 1681-4.

Zhu JF, Liu Y, Liu P, et al (2011). JAK2V617F mutation in the patients with myeloproliferative disorder and its relation with clinical characteristics. Zhongguo Shi Yan Xue Ye Xue Za Zhi, 19, 916-20. 